\title{
Prediction-Based Channel Selection Prediction in Mobile Cognitive Radio Network
}

\section{J. Jaffar ${ }^{1}$, Sharifah K.S.Yusof ${ }^{2}$, Norulhusna Ahmad ${ }^{3 *}$, Jawahir Che Mustapha ${ }^{4}$}

${ }^{1}$ Universiti Kuala Lumpur Malaysian France Institute,

Section 14, Jalan Teras Jernang, Bandar Baru Bangi, Selangor 43650, MALAYSIA

${ }^{2}$ School Of Electrical Engineering, Faculty of Engineering, Universiti Teknologi Malaysia,

Skudai, Johor Baharu, Johor, 83100, MALAYSIA.

${ }^{3}$ Razak Faculty of Technology and Informatics, Universiti Teknologi Malaysia,

Jalan Sultan Yahya Petra, Kuala Lumpur, 54100, MALAYSIA.

${ }^{4}$ Universiti Kuala Lumpur Malaysian Insitute of Information Technology,

1016 Jalan Sultan Ismail, Kuala Lumpur, 50250, MALAYSIA.

*Corresponding Author

DOI: https://doi.org/10.30880/ijie.2018.10.07.029

Received 10 August 2018; Accepted 25 November 2018; Available online 30 November 2018

\begin{abstract}
The emerging 5G wireless communications enabled diverse multimedia applications and smart devices in the network. It promises very high mobile traffic data rates, quality of service as in very low latency and improvement in user's perceived quality of experience compared to current $4 \mathrm{G}$ wireless network. This encourages the increasing demand of significant bandwidth which results a significant urge of efficient spectrum utilization. In this paper, modelling, performance analysis and optimization of future channel selection for cognitive radio network by jointly exploiting both CR mobility and primary user activity to provide efficient spectrum access is studied. The modelling and prediction method is implemented by using Hidden Markov Model algorithm. The movement of CR in wireless network yields location-varying spectrum opportunities. The current approaches in most literatures which only depend on reactive selection spectrum opportunities result of inefficient channel usages. Moreover, conventional random selection method tends to observe a higher handoff and operation delays in network performance. This inefficiency can cause continuous transmission interruptions leading to the degradation of advance wireless services. This work goal is to improve the performance of CR in terms number of handoffs and operation delays. We perform simulation on our prediction strategy with a commonly used random sensing method with and without location. Through simulations, it is shown that the proposed prediction and learning strategy can obtain significant improvements in number of handoffs and operation delays performance parameters. It is also shown that future CR location is beneficial in increasing mobile CR performance. This study also shows that the number of primary user in the network and the PU protection range affect the performance of mobile CR channel selection for all methods.
\end{abstract}

Keywords: Mobile Users, Cognitive Radio, Prediction, Spectrum Selection, Location

\section{Introduction}

Cognitive radio (CR) is employed as an opportunistic spectrum access which promotes intelligence for 5G wireless networks to provide higher capacity and a network speed of 10Gbps [1]. The need for more capacity will demand more spectrums resulting in integration of $\mathrm{CR}$ in $5 \mathrm{G}$ networks. The need of $\mathrm{CR}$ is to enable much more efficient use of the 
spectrum while intelligently adapts itself to provide the optimum communications channel. CR is expected to operate dynamically in an opportunistic way in which spectrum access is permitted only if the channel selected is detected idle. Conventionally, a CR must perform spectrum sensing periodically at each licensed channel to determine the primary user (PU) transmission states in every time slot. Literatures states that channel detection greatly depends on PU traffic pattern and sensing errors [2].

5G wireless network incorporates high mobility requirements [3], hence spectrum availability becomes location varying besides the common time-varying. This has become more complicated in providing an efficient utilization of the idle portions of the radio spectrum and resource allocation. Many studies and strategic methods had been proposed to improve the accuracy of channel selection.

\subsection{Motivation}

CR network (CRN) implements spectrum utilization by allocating low priority unlicensed users (CR) to exploit idle license channels which are used by high priority licensed users (PUs) in an opportunistic manner [4]. However, the CR must vacate the channel prior to PUs return to claim for channels access. A set of procedures called spectrum handoff will be initiated where the system will find any new vacant channel through random channel selection and spectrum sensing to resume their interrupted transmissions. It is seen that the performance of a spectrum handoff operation depends greatly on the performance of spectrum sensing. Many works studies on the decision errors provide by spectrum sensing [5]-[7], quantified in terms of false alarm and misdetection probabilities that are caused by noise and the channel impairments such as shadowing and fading. A false alarm occurs when an idle channel is sensed busy, while misdetection occurs when busy channel is sensed free. These errors will consequently lead to loss of opportunities and collision with PU in the channel respectively.

Correspondingly, many studies have focused on the spectrum handoff that depends on spectrum opportunities which is time-varying due to the random variations PUs activities [8], [9]. The QoS performance of spectrum utilization such as delay and throughput rate is being compared. In most of these works, both CR and PUs are assumed to be stationary. Evidently, in mobile CR, the spectrum opportunities change due to network topology change and the existence of transmission range. This is due to the spectrum availability exist in time and space domain at which the spectrum opportunity is due to time varying PU traffic pattern and CR or PU movement in time respectively. For spectrum opportunity in space, the CR must determine the relative position of PU transmitter. Work in [10]-[14], shows that by considering location information of the PUs or CR, CRN could optimized the channel utilization and CR throughput rate. Work in [15], [16] study the effects of the CR mobility on spectrum sensing in cognitive vehicular network has been investigated. It is seen that the PUs protection range, the CR mobility model, the network region size and the PU activity affect the detection capacity and the maximum achievable channel access probability. In [17] introduce spatial false alarm to consider the performance of spatial sensing while [18] proposed a spatial temporal sensing method to identify the channel opportunities in mobile CRN.

Note that spectrum opportunity in time and frequency domain will be determined by spectrum sensing operation. The CR can perform handoff once it confirms that it will not interfere any of the PU receivers at that time and location. The QoS in CR transmission will be degraded if frequent handoff occurs where in handoff. In handoff operation, CR will access the available channels with a cost of several handoff delay operations caused by spectrum sensing, spectrum decision, spectrum sharing, and spectrum mobility [19]. The incorrect identification of idle channel and any successive suboptimal channel selections could result in unnecessary delays and frequent handoffs. Hence, in [20]-[22] shows that proactive spectrum availability predictions will improve the channel selection process by minimizing the probability of inaccurate spectrum sensing.

This paper proposes to implement HMM learning based spectrum selection process which exploits past PU activity pattern and CR previous movements. Then, the next channel selection will be based on the prediction of next locationvarying spectrum opportunity results. This work is extended from [22] where both PU activity and location information are used for channel selection. The work employs prediction strategy using PU activity modeling to improve its channel allocation in mobile CR scenario. It shows that their prediction strategy improves further when the location of CR is being considered. In [23], [24], described several issues due to localization estimation method where high localization operation delay occurs in return of higher accuracy. Therefore, this work intends to investigate the benefits of exploitation of the past CR user movements to estimate the future CR movements using HMM algorithm. This prediction method is expected to outperform the conventional random methods which depend on reactive localization and sensing. The simulation results will show the performance improvement of the proposed method in CR networks. The work specifically deals with CR performance for number of handoff and operation delays for mobile CR user.

The rest of this paper is organized as follows. Section 2 describes the system model and proposed strategy formulation. Section 3 presents the performance comparisons and discussion of the Random with and without location information schemes. Finally, section 4 concludes this paper. 


\section{System Model and Problem Formulation}

In this section, we will describe our CRN network model. We consider a mobile CR network scenario consist of 1 $\mathrm{CR}$ and $1 \mathrm{PU}$ as shown in Fig. 1 to evaluate the performance of the proposed method

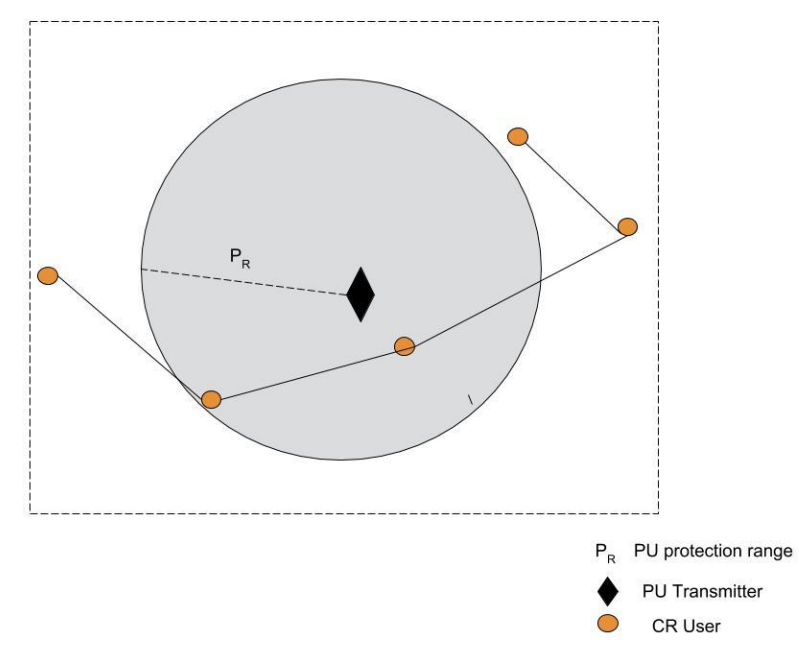

Fig. 1 Example of Moving CR and PU Scenario

\subsection{System Model Description}

In this paper, we consider a single PU base station with fixed location, $L=[x, y]$ and a single CR moving randomly inside a network area, $A$. $A$ is assumed to be square shaped for simplicity. The CR network is considered as a time slotted system, $t=\{0,1, \ldots, t+1, t+2, \ldots T\}$. CR is assume to move according to Random Way Point Mobility (RWPM) model [22]. The PU transmitter has a limited transmitting range which is defined as protection range, $P_{r}$ due to its fixed network policies and hardware constraints.

As depicted in Fig 1, in mobile network scenario, whenever a CR is outside $P_{r}$, it can use the licensed channel freely without interrupting the PUs. This is because PU has a specific transmitting range of $P_{r}$ only. It is seen that beyond the $P_{r}$, the PUs transmission signal is not reachable. While inside $P_{r}$ region, the CR can only use the licensed channels when PUs is idle similar as in static scenario in literatures. Thus, practically, the changing relative distances between PU transmitter and CR, the channel sensing and selection decision strategy can be defined to accommodate the exploitation of the extended spectrum opportunity.

\subsubsection{PU Activity Model}

This work assumes that the PU will accesses the licensed channel according to alternating renewal two state birthdeath model with birth rate, $\alpha$ and death rate, $\beta$ [16]. An ON/OFF state, $S=\{0,1\}$, denotes the occupancy of a in the channel. The channel state will alternate between ON (busy) and OFF (idle) state. The CR user is allowed to transmit only during the OFF time slots. Each channel follows the exponential ON/OFF distribution [16]. The probability density function of the time intervals for the ON/OFF states respectively satisfies:

$$
\begin{aligned}
& f_{\text {on }}(t)= \begin{cases}\beta e^{-\beta t}, & t \geq 0 \\
0, & t<0\end{cases} \\
& f_{\text {off }}(t)=\left\{\begin{array}{cc}
\alpha e^{-\alpha t}, & t \geq 0 \\
0, & t<0
\end{array}\right.
\end{aligned}
$$

The probability of channel availability is the normalized period that is available for CR. Let $P_{\text {on }}$ and $P_{\text {off }}$ denote the probability of idle channel and busy channel respectively. Then:

$$
\begin{aligned}
& P_{o n}=\frac{\beta}{\alpha+\beta} \\
& P_{o f f}=\frac{\alpha}{\alpha+\beta}
\end{aligned}
$$


PU state in the licensed channel is detected using spectrum sensing for sampling and decision operation. It is assumed that the CR is aware of its own location through GPS or other suitable localization methods [25]. Hence, this work assumes that the CR does not know the availability of a channel at each timeslot and location until they perform spectrum sensing and localization estimation. Thru spectrum sensing and localization method, the samples of previous PU state and CR locations are exploited as the samples sequence for the estimation of HMM parameters. HMM parameters estimation will be further explained in section 2.1.2. HMM training algorithm stipulates the coherency between the historical observation data which will produce the PU state model and CR movements' model. These HMM model will be used to estimate the future prediction of the spectrum opportunity.

\subsubsection{Joint Location -Channel State Prediction}

In this section, we will show formulation of spectrum selection that corresponds to the prediction of CR location in PU network region (spatial opportunity) and channel state (temporal opportunity). Then, the channel selection is performed by developing Joint Location-Channel State Prediction Access (JL-CSPA). First the HMM algorithm will be explained to do channel modeling.

HMM is a powerful statistical technique that has become increasingly popular over the last few decades. An HMM is described with respect to hidden states, observations and their model probabilities. Practically, the hidden states define the actual state of PU activity while the observation state is the data observe from the CR sensing or localization operation. Therefore, based on works [26]-[28], show that a two-state HMM can accurately predict PU activity pattern. This model relies heavily on the accuracy of the historical observation data sequence. Thus, given the investigation by these studies, HMM is chosen to be used in this work for efficient learning and prediction in both location and channel state for channel handoff.

The learning phase starts with the HMM algorithm is first fed with past observation sequence of data, $O_{k}$ (location or channel state) and trained to find the optimal HMM parameters, $\lambda$. HMM parameters $(\lambda=A, B, \pi)$ consists of state transition matrix, $a$, state observation matrix, $b$ and initial state distribution, $\pi$. State transition matrix, $a_{i, j}$ and state observation matrix, $b_{j}\left(O_{k}\right)$ is defined as:

$$
\begin{array}{ll}
a_{i j}=P\left[q_{t+1}=S_{j} \mid q_{t}=S_{i}\right] . & 1 \leq i, j \leq N \\
b_{j}\left(O_{k}\right)=P\left[O_{k} \mid q_{t}=S_{j}\right] . & 1 \leq j \leq N, 1 \leq k \leq M
\end{array}
$$

Where $N$ and $M$ are the number of hidden states and observation state respectively. The goal of training is to adjust the HMM parameters, $\lambda$, such that the PU observation sequence are best represented by the model, $P(O \mid \lambda)$. HMM training parameter is computed iteratively by using the well-known Baum-Welch Algorithm (BWA) by exploiting the coherency of the observation sequence. An HMM Prediction algorithm used in this work is similar in work [26].

\subsubsection{CR User Location Prediction}

The most trivial task in a mobile CR network to determine the spatial opportunity is to find its relative distance with the PU transmitters. Next is to initiate its transmission whenever it is convinced that it will not cause any harmful interference to any PU transmission. Given the location information to CR, the optimal available channel can be acquired effectively and the maximum performance can be achieved. Consequently, it will prevent higher number of handoff and collisions to the PU. The delay caused by handoff process can also be reduced. A spectrum opportunity in spatial definition can be modeled in another hypothesis testing problem as defined in [22]:

$$
\begin{aligned}
& \mathrm{C} 0: P_{r}<d_{i}<P_{C R} . \\
& \mathrm{C} 1: 0<d_{i}<P_{R} .
\end{aligned}
$$

where the $\mathrm{CR}, i$, distance from $\mathrm{PU}, j$, is given by $d_{i}$ as computed as:

$$
d_{i}=\left\|\left(x_{i}, y_{i}\right)-\left(x_{j}, y_{j}\right)\right\|
$$

and $P_{C R}$ is the CR specified maximum transmission range. Equation (7) defines as hypothesis C0 when CR $i$, is located outside the $P_{R}$ but within the CR maximum transmission range and resulting the channel is idle. The hypothesis C1 in equation (8) denotes when CR is located inside the $P_{R}$ and is not allowed to transmit in that channel unless the channel is sensed idle. In this work, we assume that $P_{C R}$ is within the simulation network area, $A$, specified. Thus, if a CR is located within $P_{R}$ of a busy PU on a channel, it cannot perform operation on the same channel. Spectrum sensing needs 
to be done instead to avoid PU collision. Equation (9) defines the Euclidean distance calculation to calculate the distance between the PU and CR in the network. Therefore, it shows the relative distance between the two entities determine the allowable channel utilization for CR system.

CR movement is assumed to be estimated at every timeslot and recorded in the database for HMM parameter training. The CR location coordinates, $(x(t), y(t))$ will be quantized into grid points or as defined as location index, $z(t)$ in this work. $z(t)$ is used as observation sequence, $(O(t))$ to find the estimated HMM model, $\lambda_{z}$ which describes the movement pattern of the CR. Then, by using $\lambda_{z}$, the predicted location index, $\dot{z}(t+1)$ of the CR and its location coordinates, $(\dot{x}(t+1), \dot{y}(t+1))$ can be determined from

$$
z(t+1)=a_{q(t), q(t+1)}
$$

and thus,

$$
\dot{z}(t+1)=a_{q(t+1), z(t+1)} .
$$

Following, the work will estimated, $d_{i}$, to find the so called predicted spatial state, $L=\{0,1\}$ representing spatial OFF or ON.

\subsubsection{Channel State Prediction}

In practical implementation of spectrum sensing, the sensing results will be imperfect due to various reasons such as hardware impact and noisy channels. The imperfect sensing may create errors in the form of false alarms and missed detections [29] causing misidentification of channel availability. Thus, the knowledge of the future channel states, the probability of a CR to successfully discover an idle channel is higher. Channel state estimation and prediction using HMM algorithm is proposed as in work [26] so that the CR able to sense and select target channel in an optimal order and to maximize spectrum utilization and handoff performance. HMM is chosen in this work due to [30] where it proves the validity of HMM algorithm in identifying and characterizing the pattern of observation sequence given. Thus, HMM is seen to be efficient in producing future observation data.

The method begins with the gathering of channel sensing data for a defined length of timeslot. These data are used as an observation sequence, $\mathrm{O}_{\mathrm{t}=1,2 \ldots \mathrm{T}}$ where $T$ is the length of the sampling instant. At the learning stage, the CR will use the observation sequence and estimate the HMM model parameters, $\lambda_{P}$. Then by using $\lambda_{P}$, the CR will predict the PU appearance for time slot $T+1$. In order to prevent inaccuracy in state prediction, sensing operation will be done before transmission begins.

The channel prediction will be done by finding the likelihood probabilities of $P\left[O_{T+1}=1 \mid \lambda\right]$ and $P\left[O_{T+1}=0 \mid \lambda\right]$. This is done by computing iteratively forward variable in three states as given in equation (12) to (14).

Initialization state:

$$
\Phi_{1}(i)=\pi_{i} b_{i}\left(O_{1}\right), \quad 1 \leq i \leq N
$$

Induction state:

$\Phi_{t+1}(j)=\left[\sum_{i=1}^{N} \varphi_{t}(i) \pi_{i j}\right] b_{j}\left(O_{t+1}\right), \quad 1 \leq t \leq T, 1 \leq j \leq M$

Termination state:

$P\left(O_{T+1} \mid \lambda\right)=\max \left[\sum_{i=1}^{N} \varphi_{T}(i) b_{j}\left(O_{T+1}\right)\right]$

The decision of channel state of future timeslot is determined by,

$$
Y(T+1)=\left\{\begin{array}{l}
0, P\left[O_{T+1}=1 \mid \lambda\right]>P\left[O_{T+1}=0 \mid \lambda\right] \\
1, P\left[O_{T+1}=0 \mid \lambda\right] \geq P\left[O_{T+1}=1 \mid \lambda\right]
\end{array}\right.
$$

The idle channels will be arranged in decreasing order with the highest probability of being idle.

\subsubsection{JL-CSPA Method}

In this section, the proposed JL-CSPA scheme is presented. Since the CR may move out from PU protection range, the JL-CSPA will ensure the channel utilization will not be wasted. The CR able to detect more channel opportunities beforehand and avoid frequent handoff occurs with the ability to predict CR future movement and channel states. The 
main problem addresses in this work is how the predicted CR future location and channel state can be used for channel selection. The CR will predict the channel occupancy for the next timeslot, $t+1$, by considering both location and PU activity. Thus, according to equation (5-6) and (14), the following hypotheses M0 and M1 are derived for the proposed problem as

$$
\begin{aligned}
& \text { M0 }: x_{i}(t)=\left\{\begin{aligned}
n_{i}(t) * P\left(\bar{Y}_{0}\right)_{i}, & d_{i}(t) \leq D_{S} \\
P\left(\bar{Y}_{0}\right)_{i} *\left(h_{i} s_{i}(t)+n_{i}(t)\right) \cap L_{o}, & D_{P U}<d_{i}(t) \leq D_{S} \\
P\left(\bar{Y}_{1}\right)_{i} *\left(h_{i} s_{i}(t)+n_{i}(t)\right) \cap L_{o}, & D_{P U}<d_{i}(t) \leq D_{S}
\end{aligned}\right. \\
& \text { M1 }: x_{i}(t)=P\left(\bar{Y}_{1}\right)_{i} *\left(h_{i} s_{i}(t)+n_{i}(t)\right) \cup L_{o}, \quad 0<d_{i}(t) \leq D_{P U}
\end{aligned}
$$

The CR continuous time received signal which is gained through spectrum sensing observation and is denoted as;

$$
y(t)=h * s(t)+n(t)
$$

where $y(t)$ represents the continuous received signal; $h$ is the channel gain from the PU transmitter to the CR receiver; $s(t)$ is the PU signal and $n(t)$ is the Additive White Gaussian Noise (AWGN) with variance $\sigma^{2}$ [29].

Referring to equation (16) and (17), M0 represents the channel is available for both temporally and spatially for CR and M1 as otherwise. M0 is achieved when it is either predicted idle or found that the CR is predicted moving outside $D_{p u}$ (but within $\left.D_{s}\right) . P\left(\bar{Y}_{0}\right)_{i}$ and $P\left(\overline{Y_{1}}\right)_{i}$ is the probability that the channel, $i$ is predicted idle or busy respectively.

Thus, the algorithm for the proposed work can be seen as in Fig. 2. It will start with HMM parameter training for both $\lambda_{Z}$ and $\lambda_{P}$. Then, using $\lambda_{Z}$, the $\dot{z}(t+1)$ is determined. The potential available channels will be rearranged with decreasing order of predicted idle probability of $P\left(\bar{Y}_{1}\right)_{i}$. Spectrum sensing is still required before handoff operation so that any miss prediction and sensing errors is reduced in the event. This had been showed to be beneficial as proved in work [26]. The highest potential channel will undergo sensing and allowed for handoff at the next timeslot.

Next section, we will show that this proposed method will able to reduce significant number of handoff as opposed to the conventional method with the tradeoff of successful throughput rate. The delay caused by handoff process is also shown to be reduced.

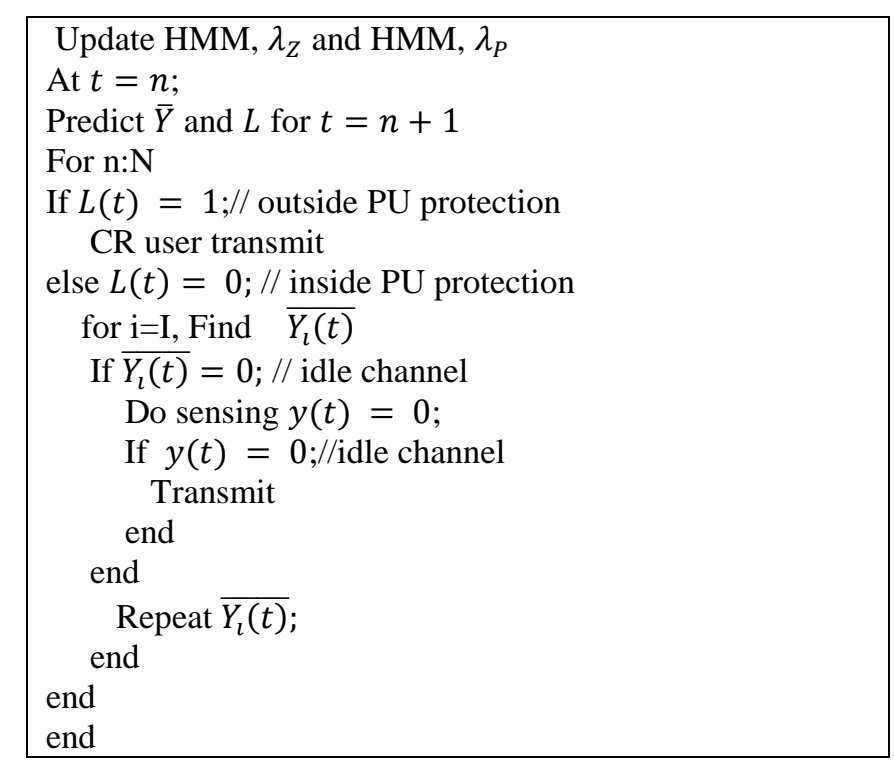

Fig. 2 JL-CSPA Algorithm

\section{Performance Evaluation}

The method of a CR to perform channel selection for its transmission has a significant impact on the performance of a CRN. We propose a prediction method that simultaneously monitors PU channels and CR movements and then utilizes the channel occupancy and CR location model described in Section 2 to predict future channel availability. By following this method, a CR is able to find future idle channel more efficiently.

The simulation system is using MATLAB. We investigate the effect of $D_{p u}$ described in section 2 . The first scenario will incorporate only $1 \mathrm{PU}$ and $1 \mathrm{CR}$ in the network area to show the improvement of JL-CSPA as $D_{p u}$ increases. Next scenario will has 2 PUs and 1 CR to further evaluates its performance when there are 2 PUs in the network. The number 
of PUs in the network brings a challenge for a mobile CR to select the best spectrum opportunity spatially and temporally. The overlapping of different PU protection range causes the CR system difficulties to find suitable channel to handoff. Moreover, CR movement may encounter different PU transmission protection, $D_{P U}$ due to many licensed users in the network area. Thus, the JL-CSPA must able to detect each $D_{P U}$ efficiently and in advance for better handoff to improve its performance. For this work, we compare the following 4 methods for channel selection:

(i) Random; this is the conventional method for channel selection where channel is randomly selected for sensing before it decides its channel allocation.

(ii) Random channel selection with location assisted (Random-LA); this method uses previous Random method but provided its location information using GPS or localization estimation (no location prediction)

(iii) Channel Selection Prediction Access (CSPA) [22]; this method uses HMM prediction method based on the PU activity only.

(iv) Proposed JL-CSPA;

\subsection{Simulation Setup}

For this work, simulation consists of 4 licensed channels in the network for CR use. The sample sensing data for HMM parameter modeling is recorded for 400 recent timeslots and simulation is done over 2000 timeslots. For the purpose of this work, the spectrum sensing and CR user localization estimation time delay are assumed as $1 \mathrm{~s}$ each. The spectrum handoff time delay, $\delta$ is $1 \mathrm{~s}$. We will assume for low SNR of received PU signal of -20dB in order to investigate our framework. The threshold for spectrum sensing is set at $\mathrm{P} f=0.1$. We placed the size of the network area as $2000 \mathrm{~m}$ $\mathrm{x} 2000 \mathrm{~m}$. The protection range of the PU on its occupied channel is varied to prove the effectiveness of this proposed method. The CR user will move according to RWPM model in the network region with uniform speed in the interval of $[5,10] \mathrm{m} / \mathrm{s}$. This work also assumes that the CR system determines its relative position given by GPS or any other positional methods and can thereby recover all the area beyond the PU protection range. Using these mutual distances between the CR and PU calculated by the CR network, the decision to select a suitable channel is perform according to the proposed method.

\subsection{Results and Discussion}

It is mentioned before that the main issue in this work is the handoff delay and the numbers of handoff for the CR to perform its communication for higher QoS. Fig. 3 shows the number of handoff as a function of the normalized PU protection range, $D_{p u}$. It is shown that the PU protection range did not affect the performance curves of both Random and CSPA. This is because these methods do not incorporate any location information in their channel selection. However, CSPA shows lower number of handoff than Random method because of it can select the best channel available in advance. However, the Random-LA and JL-CSPA method which depends on location information are affected by the PU protection range. The number of handoff increases as the PU protection range is higher. The CR will always be inside the PU protection range as the normalized $D_{p u}$ increases towards 1 . Evidently, our proposed prediction method shows highest performance which jointly predicts both channel and location, JL-CSPA.

Fig. 4 shows the average handoff delay with respect to normalized $D_{p u}$. Handoff operational delay in channel transmission operation often becomes an issue in high speed mobile applications. Evidently, the performance curve of the proposed method has shown lower average handoff delay compared to other methods. It is reasonable since in the proposed method, the CR does not need to perform spectrum sensing and localization estimation at every timeslot in order to find available channel since it already predicts its next timeslot location based on previous location and HMM parameters computed. The Random-LA observes to have the worst delay performance towards increasing $D_{p u}$. This shows how localization estimation operation may impact the handoff delay.

Fig. 5 depicts the successful channel detection with respect to PU protection range. It is seen in Fig. 5, the proposed method achieves higher successful channel detection that the Random method but obtain comparable results with CSPA. The advantages of channel state prediction with HMM learning is capable to find best available channel in any occurrence of CR user movement in the network.

Next, we investigate the proposed method for two PU in the network assuming both have the same $D_{p u}$. The speed will be varied uniformly $(5 \mathrm{~m} / \mathrm{s}$ to $10 \mathrm{~m} / \mathrm{s})$ in order to provide various conditions of changing CR locations. The normalized $D_{P U}$ is varied increasingly to provide overlapping protection range between the two PU users. Fig. 6 depicts the performance of average number of handoff. The performance of JL-CSPA is the best among all methods. Again, it shows the location information and learning method helps in efficient channel selection. Random and CSPA performance curves are constant throughout the simulation.

In Fig. 7, JL-CSPA observes the best performance for handoff delay. When $D_{P U}$ close to 1, the performance is close for both CSPA and JL-CSPA due to overlapping $D_{P U}$ for both PUs. Consequently, the Random method performs the worst while the Random-LA increases its handoff delay as the normalized $D_{P U}$ increase.

Fig. 8 illustrates the performance of normalized successful channel detection. As expected, the overall performance of the four methods increase as the normalized $D_{P U}$ increased. However, we observed that each method show slight 
difference between each other with JL-CSPA being the highest and Random only method the worst. As the normalized $D_{P U}$ increase above 0.7 , the performance gap between the Random/Random-LA and CSPA/JL-CSPA increases. This shows that during this time, the spatial domain decreases and purely depends on PU activities itself. The HMM algorithm able to exploits the correlation of PU activities at each channel from the sensing data. This investigation also shows that with two PUs being available in the network area, the channel opportunity for Random method provides a decreasing performance curves as $D_{P U}$ increase. On the contrarily, previous simulation with only one PU in the network, the successful transmission is constant regardless of $D_{P U}$ range.

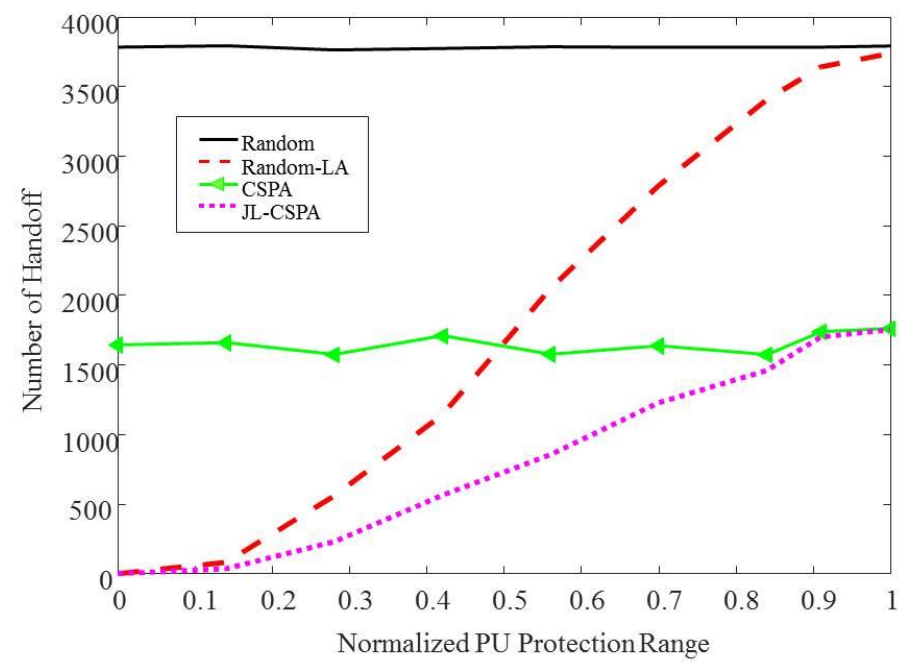

Fig. 3 The Performance of Average Number of Handoff when Normalized PU Protection Range is varied

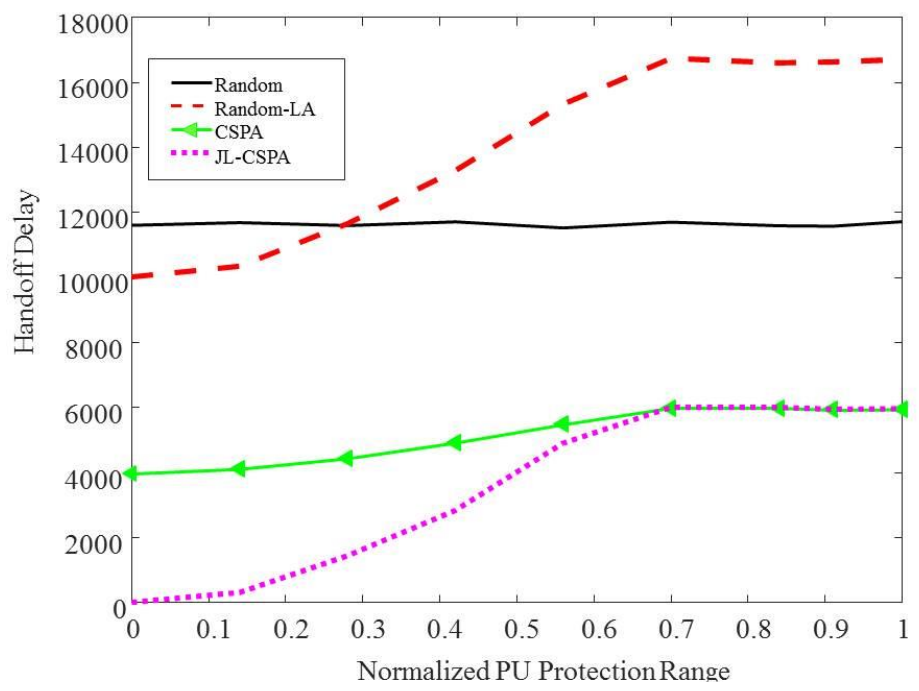

Fig. 4 The Performance of Average Handoff Delay when Normalized PU Protection Range is varied. 


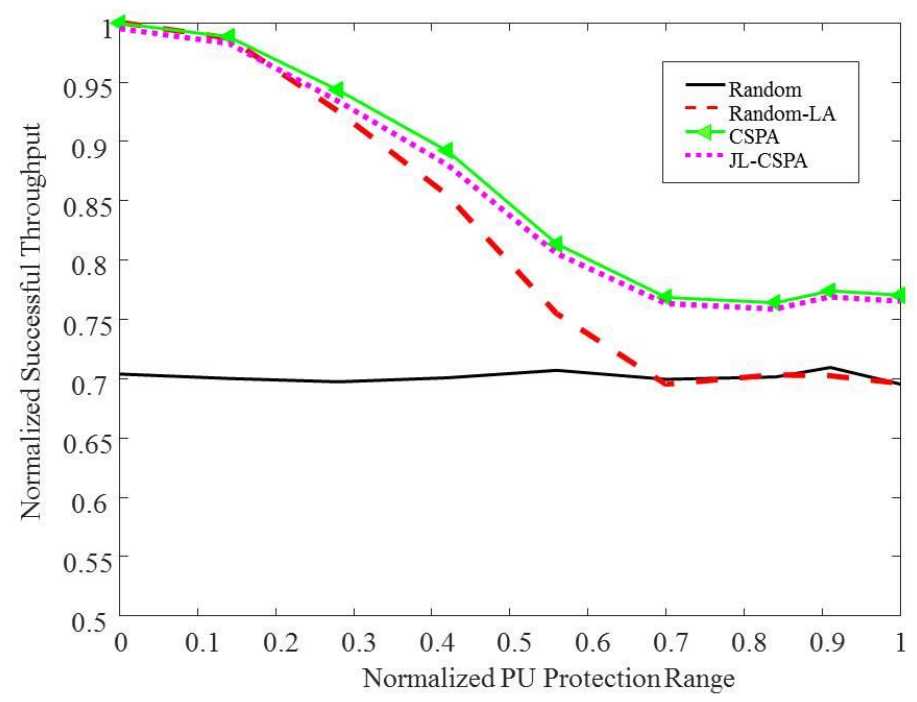

Fig. 5 The Performance of Successful Channel Detection when Normalized PU Protection Range is varied

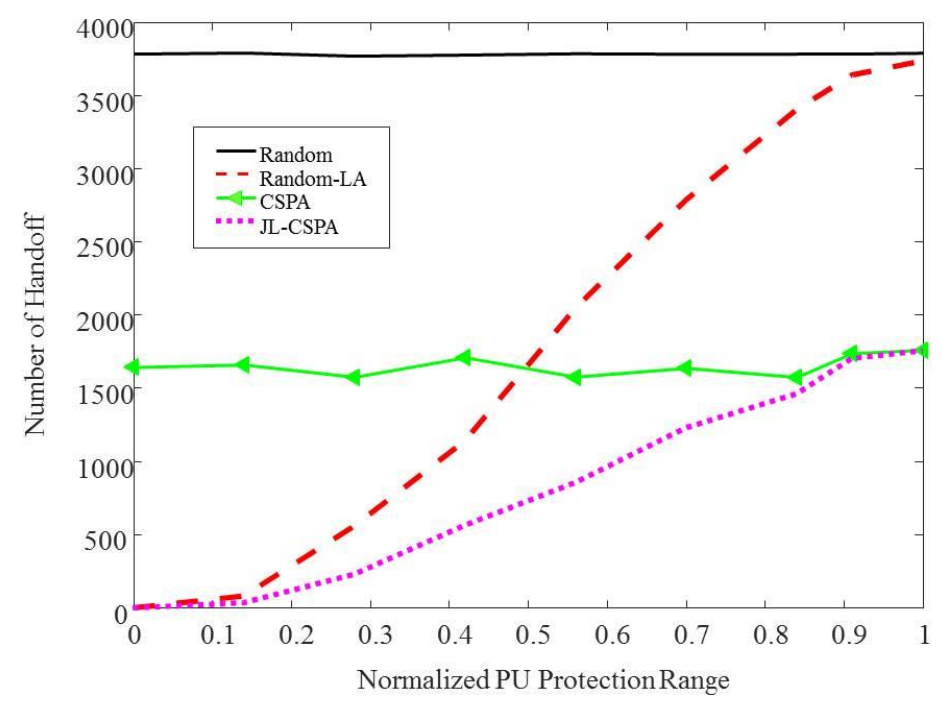

Fig. 6 The Performance of Average Number of Handoff for Two PU with Increasing PU Protection Range 


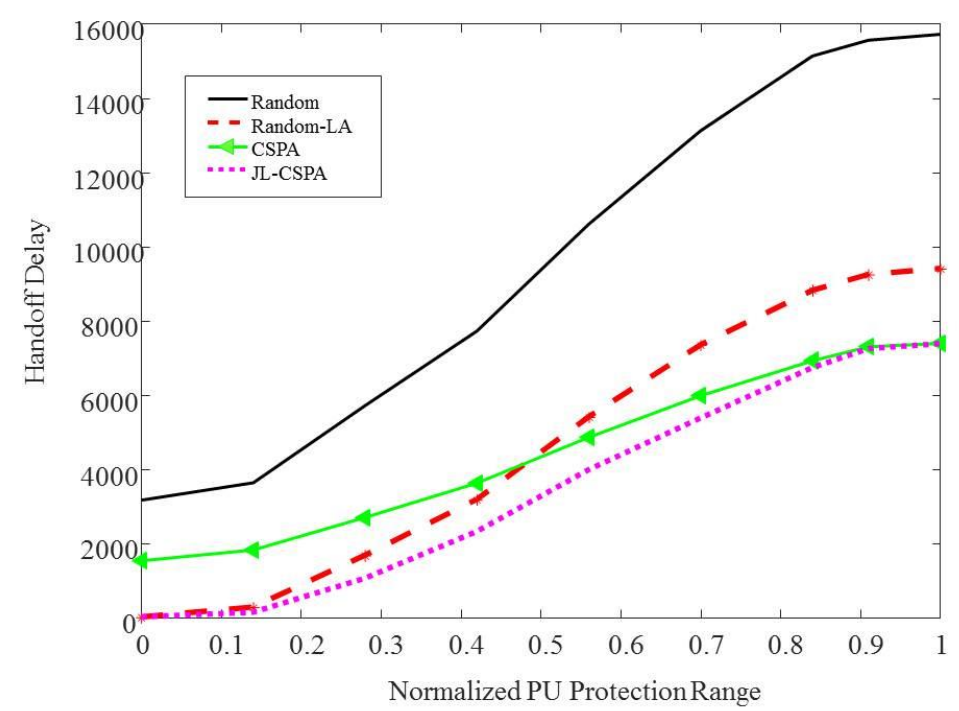

Fig. 7 The Performance of Average Handoff Delay for Two PU with Increasing PU Protection Range

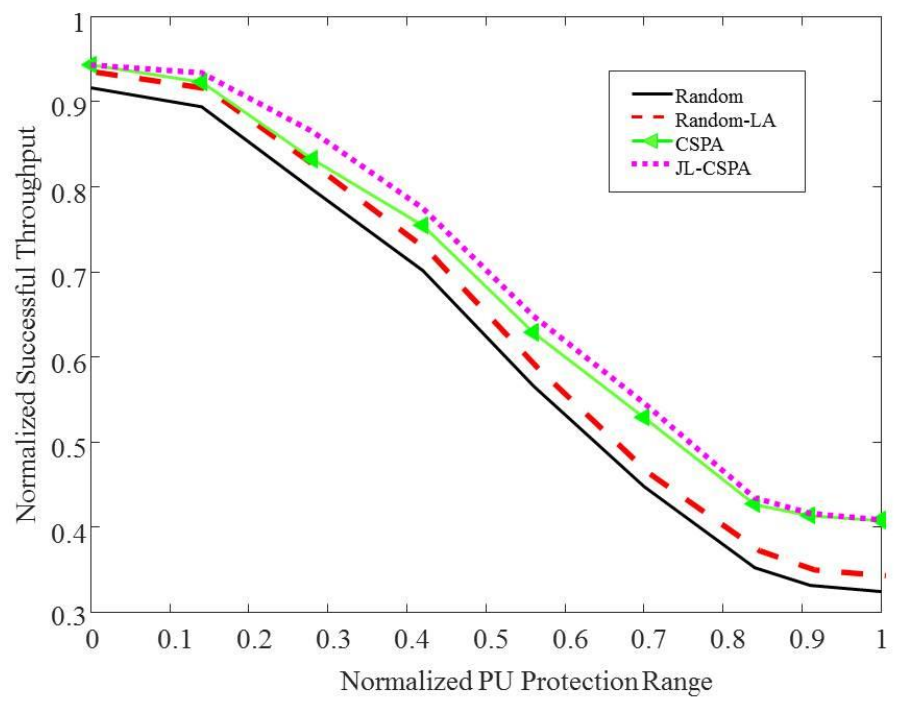

Fig. 8 The Performance of Normalized Successful Channel Detection for Two PU with Increasing PU Protection Range

\section{Conclusion}

In this paper, the JL-CSPA method is being described and investigated. The main issue of this work is to improve the CR performance in terms of operational delay and number of handoff when CR is mobile. The best available channel based on PU activity and CR location is determined in advance by using the HMM learning algorithm. The JL-CSPA has shown promising result to improve handoff operation performance by reducing the need to perform unnecessary sensing and localization in every timeslot. Hence, higher capacity and efficient bandwidth utilization can be achieved. We also have shown the benefits of HMM learning and prediction which able to estimate the future CR location and channel idle state in the network. Numerical results have evidently shown the efficiency of implementing the proposed method in mobile cognitive radio networks. Future work is to incorporate more mobile CR in the area network to evaluate the spectrum selection efficiency. 


\section{Acknowledgment}

The authors would like to express their gratitude to UTM Razak School for the financial support offered through the project grant corresponding to DPUTMRAZAK Grant (No: PY/2017/00927).

\section{References}

[1] S. Sasipriya and R. Vigneshram, "An overview of cognitive radio in 5g wireless communications," in 2016 IEEE International Conference on Computational Intelligence and Computing Research, ICCIC 2016, 2017.

[2] M. T. Masonta, M. Mzyece, and N. Ntlatlapa, "Spectrum Decision in Cognitive Radio Networks: A Survey," ieeexplore.ieee.org, vol. 15, no. 3, pp. 1-20, Jan. 2013.

[3] P. Fan, J. Zhao, and I. Chih-Lin, "5G high mobility wireless communications: Challenges and solutions," China Communications, vol. 13. pp. 1-13, 2016.

[4] L. Gavrilovska, V. Atanasovski, I. Macaluso, and L. A. Dasilva, "Learning and reasoning in cognitive radio networks," IEEE Commun. Surv. Tutorials, vol. 15, no. 4, pp. 1761-1777, 2013.

[5] D. M. M. Plata and Á. G. A. Reátiga, "Evaluation of energy detection for spectrum sensing based on the dynamic selection of detection-threshold," Procedia Eng., vol. 35, pp. 135-143, Jan. 2012.

[6] N. Nguyen-Thanh and I. Koo, "A cluster-based selective cooperative spectrum sensing scheme in cognitive radio," EURASIP J. Wirel. Commun. Netw., vol. 2013, no. 1, p. 176, 2013.

[7] E. Pei, J. Li, and F. Cheng, "Sensing-throughput tradeoff for cognitive radio networks with additional primary transmission protection,” J. Comput. Inf. Syst., vol. 10, pp. 3767-3773, 2013.

[8] L. Zhang, T. Song, M. Wu, J. Guo, D. Sun, and B. Gu, "Modeling for spectrum handoff based on secondary users with different priorities in cognitive radio networks," in 2012 International Conference on Wireless Communications and Signal Processing, WCSP 2012, 2012, pp. 1-6.

[9] S. Zahed, I. Awan, and G. Min, "Prioritized proactive scheme for spectrum handoff decision in cognitive radio networks," in Proceedings - 2012 7th International Conference on Broadband, Wireless Computing, Communication and Applications, BWCCA 2012, 2012, pp. 335-341.

[10] A. Vizziello, S. Kianoush, L. Favalli, and P. Gamba, "Location based routing protocol exploiting heterogeneous primary users in cognitive radio networks," in IEEE International Conference on Communications, 2013, pp. 28902894.

[11] J. V. Subramanian and A. K. Sadiq, "Mobile Location Prediction Methods - A Survey,” Int. J. Softw. Web Sci., vol. 7(1), pp. 109-112, 2014.

[12] H. Hu, H. Zhang, and N. Li, "Location-information-assisted joint spectrum sensing and power allocation for cognitive radio networks with primary-user outage constraint," IEEE Trans. Veh. Technol., vol. 65, no. 2, pp. 658672, 2016.

[13] R. R. Thomas, S. D. Barnes, and B. T. Maharaj, "TOA location estimation based on cognitive radio channel occupancy prediction," 2012 IEEE 8th Int. Conf. Wirel. Mob. Comput. Netw. Commun., pp. 733-738, 2012.

[14] H. Celebi and H. Arslan, "Utilization of location information in cognitive wireless networks," IEEE Wirel. Commun. Mag., vol. 14, no. 4, pp. 6-13, 2007.

[15] X. Liu, Z. Zeng, C. Guo, and S. Zhu, "Performance analysis of spatial-temporal spectrum sensing for cognitive vehicular network," in Proceedings of the 3rd ACM Workshop on Mobile Sensing, Computing and Communication, 2016, pp. 1-6.

[16] A. S. Cacciapuoti, M. Caleffi, L. Paura, and M. A. Rahman, "Channel Availability for mobile cognitive radio networks," J. Netw. Comput. Appl., vol. 47, no. 2015, pp. 1-16, 2014.

[17] W. Han, J. Li, S. Member, Z. Li, J. Si, and Y. Zhang, "Spatial False Alarm in Cognitive Radio Network," IEEE Trans. SIGNAL Process., vol. 61, no. 6, pp. 1375-1388, 2013.

[18] T. Do and B. Mark, "Joint spatial-temporal spectrum sensing for cognitive radio networks," Veh. Technol. IEEE Trans., pp. 5-10, 2010.

[19] X. Xing, T. Jing, W. Cheng, X. Zhao, T. Jing, and W. Cheng, "Spectrum Prediction in Cognitive Radio Networks," Wirel. Commun. IEEE, vol. 20, no. 2, pp. 90-96, 2013.

[20] V. Balaiah, "Modeling and Analysis for Proactive Spectrum Handoff in Cognitive Radio Adhoc Networks with Improved Throughput and Security," Int. J. Wired Wirel. Commun., vol. 2, no. 2, pp. 5-9, 2014.

[21] L. C. Wang and C. W. Wang, "Spectrum handoff for cognitive radio networks: Reactive-sensing or proactivesensing?," in Conference Proceedings of the IEEE International Performance, Computing, and Communications Conference, 2008, pp. 343-348.

[22] J. Jaffar, S. Ksyusof, N. Ahmad, and J. C. Mustapha, "Location assisted proactive channel in heterogeneous cognitive radio network," J. Telecommun. Electron. Comput. Eng., vol. 8, no. 3, 2016.

[23] M. Farooq-i-Azam and M. Ayyaz, "Location and Position Estimation in Wireless Sensor Networks," J. Immunol., pp. 179-214, 2016. 
[24] S. Halder and A. Ghosal, "A survey on mobility-assisted localization techniques in wireless sensor networks," J. Netw. Comput. Appl., vol. 60, no. 2016, pp. 82-94, 2016.

[25] L. Zhang, K. Zeng, and P. Mohapatra, "Opportunistic Spectrum Scheduling for Mobile Cognitive Radio Networks in White Space," in 2011 IEEE Wireless Communications and Networking Conference, WCNC 2011, 2011, pp. 844-849.

[26] J. Jasrina, S. K. S. Yusof, and M. A. M. Izhar, "Intelligent Selective Spectrum Access in Cognitive Radio Networks," J. Artif. Intell., vol. 9, no. 4, pp. 65-71, 2016.

[27] M. S. El Din, M. El-Tarhuni, K. Assaleh, and S. Kiranyaz, “An HMM-based spectrum access algorithm for cognitive radio systems," 2015 Int. Conf. Inf. Commun. Technol. Res. ICTRC 2015, no. 3, pp. 116-119, 2015.

[28] S. D. Barnes and B. T. Maharaj, "Prediction based channel allocation performance for cognitive radio," Int. J. Electron. Commun. (AË̈)., vol. 68, no. 4, pp. 336-345, Oct. 2014.

[29] W.-Y. Y. Lee, Ian. F. Akyildiz, I. F. Akyildiz, and Ian. F. Akyildiz, "Optimal Spectrum Sensing Framework for Cognitive Radio Networks," IEEE Trans. Wirel. Commun., vol. 7, no. 10, pp. 3845-3857, 2008.

[30] C. Ghosh, C. Cordeiro, D. P. Agrawal, and M. B. Rao, "Markov chain existence and Hidden Markov models in spectrum sensing," in 2009 IEEE International Conference on Pervasive Computing and Communications, 2009, pp. 1-6. 